\title{
SUM LIST EDGE COLORINGS OF GRAPHS
}

\author{
Arnfried Kemnitz \\ Massimiliano Marangio \\ Computational Mathematics \\ Technical University Braunschweig \\ Pockelsstraße 14, 38106 Braunschweig, Germany \\ e-mail: a.kemnitz@tu-bs.de, m.marangio@tu-bs.de \\ AND \\ Margit Voigt \\ Faculty of Information Technology and Mathematics \\ University of Applied Sciences \\ Friedrich-List-Platz 1, 01069 Dresden, Germany \\ e-mail: mvoigt@informatik.htw-dresden.de
}

\begin{abstract}
Let $G=(V, E)$ be a simple graph and for every edge $e \in E$ let $L(e)$ be a set (list) of available colors. The graph $G$ is called $L$-edge colorable if there is a proper edge coloring $c$ of $G$ with $c(e) \in L(e)$ for all $e \in E$. A function $f: E \rightarrow \mathbb{N}$ is called an edge choice function of $G$ and $G$ is said to be $f$-edge choosable if $G$ is $L$-edge colorable for every list assignment $L$ with $|L(e)|=f(e)$ for all $e \in E$. Set $\operatorname{size}(f)=\sum_{e \in E} f(e)$ and define the sum choice index $\chi_{s c}^{\prime}(G)$ as the minimum of size $(f)$ over all edge choice functions $f$ of $G$.

There exists a greedy coloring of the edges of $G$ which leads to the upper bound $\chi_{s c}^{\prime}(G) \leq \frac{1}{2} \sum_{v \in V} d(v)^{2}$. A graph is called sec-greedy if its sum choice index equals this upper bound.

We present some general results on the sum choice index of graphs including a lower bound and we determine this index for several classes of graphs. Moreover, we present classes of sec-greedy graphs as well as all such graphs of order at most 5 .
\end{abstract}

Keywords: sum list edge coloring, sum choice index, sum list coloring, sum choice number, choice function, line graph.

2010 Mathematics Subject Classification: 05C15. 


\section{REFERENCES}

[1] A. Berliner, U. Bostelmann, R.A. Brualdi and L. Deaett, Sum list coloring graphs, Graphs Combin. 22 (2006) 173-183.

doi:10.1007/s00373-005-0645-9

[2] F. Harary, Graph Theory (Addison-Wesley, Reading, MA, 1969).

[3] B. Heinold, Sum list coloring and choosability (Ph.D. Thesis, Lehigh University, 2006).

[4] G. Isaak, Sum list coloring $2 \times n$ arrays, Electron. J. Combin. 9 (2002) \# N8.

[5] G. Isaak, Sum list coloring block graphs, Graphs Combin. 20 (2004) 499-506. doi:10.1007/s00373-004-0564-1

[6] A. Kemnitz, M. Marangio and M. Voigt, Bounds for the sum choice number, submitted, 2015.

[7] A. Kemnitz, M. Marangio and M. Voigt, Sum list colorings of small graphs, preprint, 2015.

[8] A. Kemnitz, M. Marangio and M. Voigt, Sum list colorings of wheels, Graphs Combin. 31 (2015) 1905-1913.

doi:10.1007/s00373-015-1565-y

[9] E. Kubicka and A.J. Schwenk, An introduction to chromatic sums, in: A.M. Riehl, (Ed.), Proc. ACM Computer Science Conference (Louisville) (ACM Press, New York, 1989) 39-45.

doi:10.1145/75427.75430

[10] M.A. Lastrina, List-coloring and sum-list-coloring problems on graphs (Ph.D. Thesis, Iowa State University, 2012).

Received 18 December 2014

Revised 2 November 2015

Accepted 2 November 2015 